\title{
Systematic neutron guide misalignment for an accelerator-driven spallation neutron source
}

\author{
C. Zendler ${ }^{1, *}$ and P. M. Bentley ${ }^{1,2}$ \\ ${ }^{1}$ European Spallation Source ESS AB, Box 176, 22100 Lund, Sweden \\ ${ }^{2}$ University of Uppsala, Uppsala 75120 , Sweden
}

(Received 2 November 2015; published 4 August 2016)

\begin{abstract}
The European Spallation Source (ESS) is a long pulse spallation neutron source that is currently under construction in Lund, Sweden. A considerable fraction of the 22 planned instruments extend as far as $75-150 \mathrm{~m}$ from the source. In such long beam lines, misalignment between neutron guide segments can decrease the neutron transmission significantly. In addition to a random misalignment from installation tolerances, the ground on which ESS is built can be expected to sink with time, and thus shift the neutron guide segments further away from the ideal alignment axis in a systematic way. These systematic errors are correlated to the ground structure, position of buildings and shielding installation. Since the largest deformation is expected close to the target, even short instruments might be noticeably affected. In this study, the effect of this systematic misalignment on short and long ESS beam lines is analyzed, and a possible mitigation by overillumination of subsequent guide sections investigated.
\end{abstract}

DOI: 10.1103/PhysRevAccelBeams.19.083501

\section{INTRODUCTION}

Neutron scattering instruments employ neutron guides to transport neutrons from a source to an experimental area, which are often separated by distances between 20 and $100 \mathrm{~m}$ - and in the case of European Spallation Source (ESS) even up to $150 \mathrm{~m}$. Great effort has been invested into the design of neutron guide geometries to optimize the beam transport over large distances (e.g. [1-4]), usually assuming ideal geometries.

Deviations introduced in real instruments in the manufacturing or installation process are less exhaustively studied, with focus on small and randomly distributed effects. Simulations of a polygonal approximation of an elliptic guide [5] identified the segment length acceptable in different parts of an ellipse to avoid multiple reflections entailing a loss in transmission and an inhomogeneous phase space, but still assumed perfect alignment and no fabrication errors.

The impact of random guide segment misalignment on the neutron transmission was estimated by measurement of a miniature short guide with a large displacement of one segment and extrapolation to a long guide with small displacements of many segments in [6]. A 5\% loss was predicted over 50-60 m for an alignment error of $8 \mu \mathrm{m}$ and

\footnotetext{
*Corresponding author. carolin.prabhu@ife.no

Published by the American Physical Society under the terms of the Creative Commons Attribution 3.0 License. Further distribution of this work must maintain attribution to the author(s) and the published article's title, journal citation, and DOI.
}

a fabrication uncertainty of $4 \mu \mathrm{m}$ [7], which corresponds to less than $0.1 \%$ of the guide extension. This was confirmed after the beginning of operations [8].

A simulation study of different loss mechanisms in supermirror neutron guides [9] shows that a loss caused by waviness of the supermirror substrate can be compensated by usage of a slightly higher $m$ value, while dimension errors and gaps can lead to a significant transmission decrease. The amount of misalignment which is deemed acceptable corresponds to about $0.05 \%$ of the smaller side length of the rectangular reference guide.

Another simulation of angular as well as positional misalignment for a specific instrument [10] comes to similar conclusions with an acceptable spatial offset smaller than about $0.08 \%-0.2 \%$ of the variable guide width in horizontal and vertical dimension, and a maximum angular deviation of $\pm 0.02^{\circ}-0.1^{\circ}$.

The alignment precision of guide segments at ESS has been estimated to $50 \mu \mathrm{m}$ [11], a value also reported at NIST [12]. This corresponds to $0.17 \%$ of a $3 \mathrm{~cm}$ wide guide, a width which is planned for two of the first three instruments accepted at ESS [13,14], hence the results of the previous studies imply that the impact of this random misalignment can already be expected to be visible. Indeed an extensive simulation study of random misalignment in straight, curved, ballistic and multichannel guides with lengths up to $150 \mathrm{~m}$ shows severe beam losses for long guides with a small cross section [15]. An additional systematic shift of maximally $3 \mathrm{~mm}$ over ten years was translated into an additional random contribution of $150 \mu \mathrm{m}$, assuming a realignment every six months and ignoring the systematic nature. 


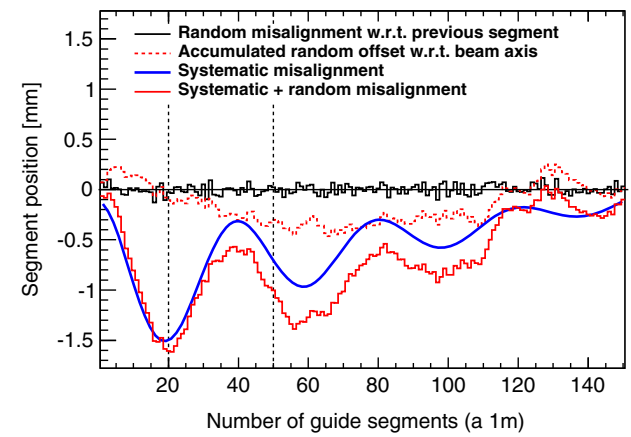

(a)

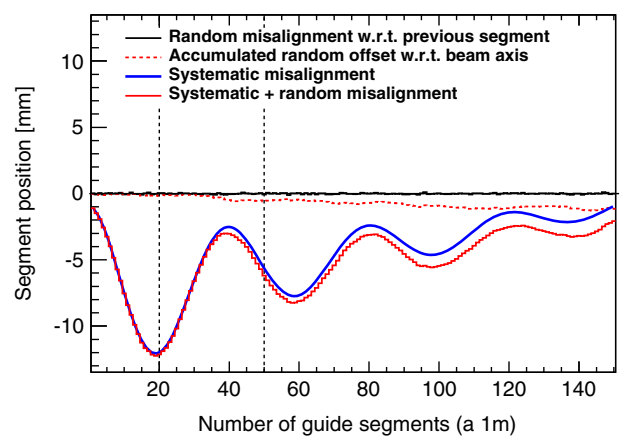

(b)

FIG. 1. Position of the beam line segments as a function of the number of guide segments, two examples of the combination of systematic and random neutron guide misalignment: the black line shows a random misalignment of $50 \mu \mathrm{m}$ with respect to the previous guide segment, the dotted red line the accumulated random misalignment with respect to the nominal guide axis. The blue line shows the systematic misalignment which is added to the random component to give the total misalignment with respect to the nominal guide axis, shown as solid red line. Guide segments are $1 \mathrm{~m}$ long. Note the different $y$ axis scale to compare these examples. $1.5 \mathrm{~mm}$ (a) and $12 \mathrm{~mm}$ (b) maximal systematic misalignment.

More recently, additional data on the ground at the ESS site reveals additional maximal systematic misalignment, which has now increased to $(3+3) \mathrm{mm}=6 \mathrm{~mm}$ (elastic + creep) [16]. This is caused by elastic deformations in the piling as well as irreversible short and long term deformations in the whole pile-ground system. While the first component has been measured to some extent by the ESS Conventional Facilities Directorate, the latter two effects involve extrapolation in time with large uncertainties [16].

It is therefore important to evaluate the expected transmission loss for a large range of guide element systematic misalignments, with a realistic model, and develop a strategy to prevent performance issues of the instruments.

\section{THEORETICAL MODEL OF SYSTEMATIC MISALIGNMENT}

The ground on which ESS is built is expected to sink over time, and more so in regions where heavy shielding is installed. Experience from SNS [17] allows us to construct an empirical model of vertical guide misalignment based on a damped cosine shape and a sloped line. Since the common shielding features are mirrored at most spallation sources-i.e. large, heavy monolith, heavy instrument stations placed at mixed distances, and a small number of independent floor slabs - we can expect a similar shape at the ESS, but with varying amplitude. We therefore model the vertical offset $\Delta z$ caused by systematic misalignment with the following function:

$$
\Delta z=-A+A \cos \left(2 \pi \frac{x}{x_{p}}\right) e^{-B x}+\frac{A}{L}\left(x-\frac{x_{p}}{2}\right) .
$$

The amplitude $A$ is calculated such that the first minimum corresponds to the maximal expected misalignment, which is tested for several values due to the large uncertainty in the estimate. The period length $x_{p}$ is set to $40 \mathrm{~m}$ such that the end of the bunker at $30 \mathrm{~m}$ is at $3 / 4$ of the cosine function.
The dampening reduces the oscillation amplitude by half within one period, i.e. the dampening factor $B$ is set such that $e^{-B x_{p}}=0.5$. The inclination of the cosine's axis is chosen such that it cuts the nominal beam line axis at $L=150 \mathrm{~m}$. Since the most realistic values can only be estimated, in particular for the dampening and the axis tilt, the effect of a variation in these parameters on the results is studied in Sec. V.

Function (1) is plotted using blue lines in Fig. 1 for the smallest and largest amplitude. The total misalignment including systematic and random contributions can be seen by the red solid line. A random misalignment of $50 \mu \mathrm{m}$ is assumed and taken with respect to the previous guide segment (black line), so the total random misalignment with respect to the nominal beam axis can add up to much more than $50 \mu \mathrm{m}$ (red dotted line). As expected, the total misalignment in the case of a $1.5 \mathrm{~mm}$ ground movement can vary greatly, while it is dominated by the systematic contribution otherwise.

\section{INSTRUMENT PERFORMANCE WITH SYSTEMATIC MISALIGNMENT}

The effect of including both random and systematic guide misalignment is investigated in the following section, for several different values of total maximal ground movement [the maximal amplitude of Eq. (1)] of 1.5, 3.0, 6.0 and $12 \mathrm{~mm}$.

Each systematic misalignment amplitude has been studied with five different generations of random misalignment -i.e. using a different random number seed - to determine the spread of the transmission loss to be expected. The random misalignment is simulated as a shift with respect to the previous section by an amount determined by a Gaussian random number around $0 \mu \mathrm{m}$ with standard deviation $50 \mu \mathrm{m}$. For each segment, such a random number is added to the systematic misalignment calculated with function (1) as $\Delta z\left(x_{i}\right)-\Delta z\left(x_{i-1}\right)$. 
TABLE I. Parameters used in the VITESS simulations.

\begin{tabular}{ll}
\hline \hline Simulation parameter & \multicolumn{1}{c}{ Value } \\
\hline Source module & ESS_2012 \\
Moderator model & 2013 Schoenfeldt, cold \\
Wavelength band & $1-10 \AA$ \\
Time period & $0-10 \mathrm{~ms}$ \\
Divergence range & Defined by guide entry \\
Distance moderator to guide entry & $2 \mathrm{~m}$ \\
Guide cross sections & $3 \times 3 \mathrm{~cm}^{2}, 10 \times 10 \mathrm{~cm}^{2}$ \\
Guide segment length & $1 \mathrm{~m}$ \\
Guide length & $20 \mathrm{~m}, 150 \mathrm{~m}$ \\
Supermirror coating & 3 \\
Random misalignment & Gaussian with $\mu=0 \mu \mathrm{m}$, \\
& $\sigma=50 \mu \mathrm{m}$ \\
Systematic misalignment $\Delta z_{\text {syst }}$ & $1.5 \mathrm{~mm}, 3.0 \mathrm{~mm}, 6.0 \mathrm{~mm}$, \\
& $12.0 \mathrm{~mm}$ \\
Systematic amplitude & $0.88,1.76,3.51,7.03$ \\
$\quad$ factor A $\left(\Delta z_{\text {syst }}\right)$ & $0.017 \mathrm{~m}^{-1}$ \\
Systematic dampening factor B & $40 \mathrm{~m}$ \\
Systematic period factor $x_{p}$ & \\
\hline \hline
\end{tabular}

Simulations have been performed with the Monte Carlo ray-tracing software VITESS $[18,19]$, using $10^{9}$ trajectories such that the statistical uncertainty on the intensity at the guide exit is well below 1\% in all cases. The simulated source is the ESS Technical Design Report [20] cold moderator, but since results are given as relative transmissions, they are independent of the source spectrum. The divergence band simulated is defined by the guide entry width and height in a $2 \mathrm{~m}$ distance from the source moderator. Gravity effects are included in the simulation. The parameters of the simulation are summarized in Table I.

Figure 2(a) shows the transmission with misalignment divided by the transmission without any misalignment for a
$20 \mathrm{~m}$ long guide with a constant cross section of $3 \times 3 \mathrm{~cm}^{2}$ and with $1 \mathrm{~m}$ long guide segments, using a supermirror coating of $m=3$. Segments are parallely shifted by the amount of calculated misalignment at their position, they are not tilted since an angular offset corresponding to a certain spatial displacement has been shown to have a minor effect compared to the spatial shift itself [15]. Systematic misalignment is applied to the vertical direction, random misalignment both horizontally and vertically.

As already seen in [9], no wavelength dependence in the transmission losses was observed for straight neutron guides with misalignment. The statistical fluctuations in the very short wavelength range are due to the fact that the cold moderator was used. In the following, the intensity at the guide exit is evaluated for $\lambda \geq 1 \AA$.

A $1.5 \mathrm{~mm}$ ground movement does not necessarily decrease the transmission significantly, because the random misalignment can contribute to the same order of magnitude. Simulations with different random misalignments show examples where the transmission with $1.5 \mathrm{~mm}$ systematic misalignment on top of the random one is equal or even slightly higher than with the random misalignment alone, but also examples where it is significantly lower. The mean relative transmission with $1.5 \mathrm{~mm}$ systematic misalignment is 0.93 compared to 0.94 with only random misalignment in the $20 \mathrm{~m}$ long guide. For guides that are $150 \mathrm{~m}$ long, $1.5 \mathrm{~mm}$ systematic misalignment produces a transmission of 0.63 , compared to 0.67 when only random misalignment is considered.

For a ground movement of $3 \mathrm{~mm}$ or more, a more significant reduction of the guide transmission is observed. The average relative transmission is given in Table II, and illustrated in Fig. 2(b) together with the relative transmission with systematic misalignment alone (i.e. without

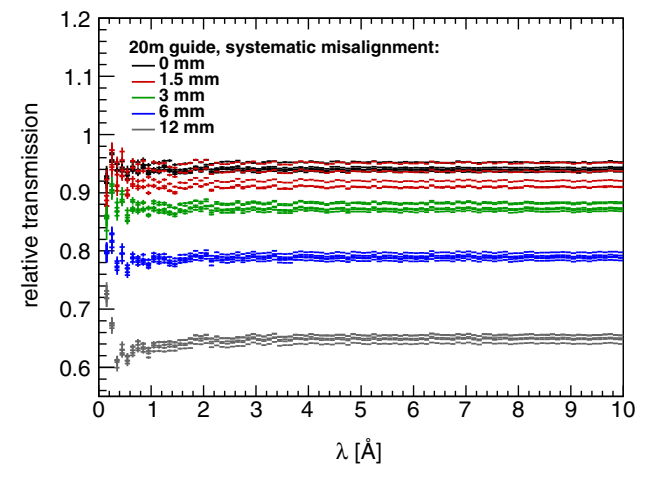

(a)

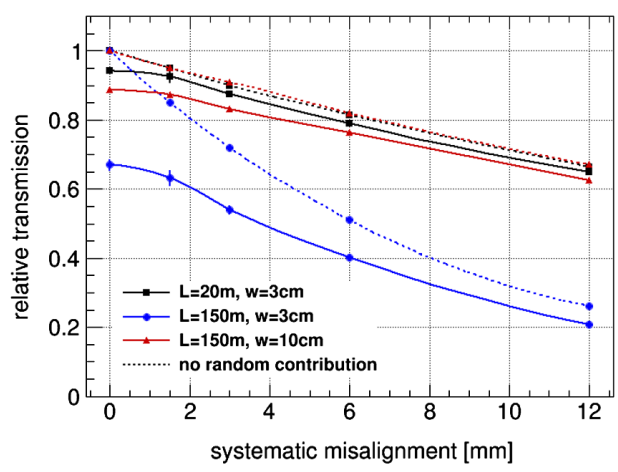

(b)

FIG. 2. Relative transmission with respect to perfectly aligned guide with different amounts of a systematic vertical misalignment. Guide sections are $1 \mathrm{~m}$ long. (a) Relative transmission of a $20 \mathrm{~m}$ guide with $3 \times 3 \mathrm{~cm}^{2}$ cross section as a function of wavelength, with systematic misalignment as well as $50 \mu \mathrm{m}$ random misalignment both horizontally and vertically. Error bars show the statistical uncertainty of the simulation only, the statistical uncertainty of the random misalignment is exemplary indicated by five different simulations with five different random numbers for the random misalignment. (b) Relative transmission as a function of maximal systematic misalignment with (solid) and without (dotted) additional $50 \mu \mathrm{m}$ random misalignment, for different guide lengths $L$ and widths $w$. Systematic misalignments of 0, 1.5, 3.0, 6.0 and $12.0 \mathrm{~mm}$ are simulated; the lines are a guide for the eye. 
TABLE II. Mean relative transmission \%, with systematic and random misalignment, averaged over five random misalignments, for different guide lengths $L$ and widths $w$.

\begin{tabular}{|c|c|c|c|c|c|}
\hline Maximum systematic misalignment & $0 \mathrm{~mm}$ & $1.5 \mathrm{~mm}$ & $3.0 \mathrm{~mm}$ & $6.0 \mathrm{~mm}$ & $12.0 \mathrm{~mm}$ \\
\hline$L=20 \mathrm{~m}, w=3 \mathrm{~cm}$ & $94.2 \pm 0.6$ & $92.5 \pm 1.6$ & $87.5 \pm 0.6$ & $78.9 \pm 0.5$ & $64.9 \pm 0.6$ \\
\hline$L=150 \mathrm{~m}, w=3 \mathrm{~cm}$ & $67.0 \pm 1.5$ & $63.3 \pm 2.2$ & $54.0 \pm 1.3$ & $40.2 \pm 0.5$ & $20.8 \pm 0.3$ \\
\hline$L=150 \mathrm{~m}, w=10 \mathrm{~cm}$ & $88.8 \pm 0.6$ & $87.3 \pm 0.9$ & $83.2 \pm 0.6$ & $76.3 \pm 0.3$ & $62.5 \pm 0.2$ \\
\hline
\end{tabular}

any random misalignment). Note that the transmission loss depends on the misalignment relative to the guide width; while a $3 \mathrm{~cm}$ wide guide is not unusual for $20 \mathrm{~m}$ long instrument (e.g. LoKI $3 \times 3 \mathrm{~cm}^{2}$ [13]), longer beam lines often have a ballistic shape with larger extension in at least parts of the beam line, therefore the mean loss for a $150 \mathrm{~m}$ long $10 \times 10 \mathrm{~cm}^{2}$ constant guide is given as well. Long instruments will most likely suffer transmission losses lying in between the given range of values, depending on the height of the guide.

The relative transmission loss does not depend on the divergence to be transported: simulations of the $150 \mathrm{~m}$ long $3 \times 3 \mathrm{~cm}^{2}$ guide with a supermirror coating of $m=1$ and $m=5$ instead of $m=3$ showed no difference in the relative loss by systematic misalignment compared to the perfectly aligned guide with the same $m$-coating.

\section{MITIGATION BY OVERILLUMINATION}

Overillumination is a mitigation strategy that can prevent beam losses from misalignment by decreasing the cross section of subsequent guide pieces. If a misalignment of $\pm x$ is expected, guide segments are overilluminated by $2 x$. This causes a certain amount of beam loss compared to a perfect guide as well, and as shown in a previous study of purely random misalignment [15], the gain from overillumination is only larger than the loss it entails for long guides with small guide cross sections. A large relative misalignment is given in the case of the systematic ground movement envisaged for ESS, therefore a vertical overillumination is studied in the following in combination with different amounts of systematic vertical misalignment.

As an example, the maximum systematic shift promised by conventional facilities shall now be assumed to be $6 \mathrm{~mm}$, which is indeed the case at the time of writing. If a long beam line is thought to be made of four sections up to 30 , 60,100 and $150 \mathrm{~m}$ from the source, this translates into a medium relative misalignment between $1 \mathrm{~m}$ long segments of roughly 250,150, 100 and $50 \mu \mathrm{m}$, respectively. Figure 3 shows the relative transmission of a guide employing overillumination of twice those values between each segment pair, depending on their location, as a function of an actual maximum systematic misalignment between 0 and $12 \mathrm{~mm}$ (blue line, round markers). For comparison, the relative transmission without overillumination is shown again (black dotted line, rectangular markers).

In the $150 \mathrm{~m}$ long guide, the loss from pure systematic misalignment of $50 \%$ can be reduced to about $35 \%$ if the maximum systematic misalignment is indeed $6 \mathrm{~mm}$. This $15 \%$ gain compared to no overillumination increases slightly for stronger ground movements. If however the ground is more stable than expected, the gain from overillumination is smaller and reaches zero by half the expected misalignment. An additional 30\% loss from this kind of overillumination is certain if no systematic misalignment is present at all, which should be the case directly after deployment.

In order to avoid such an unacceptable performance drop, the overillumination can be employed in the first

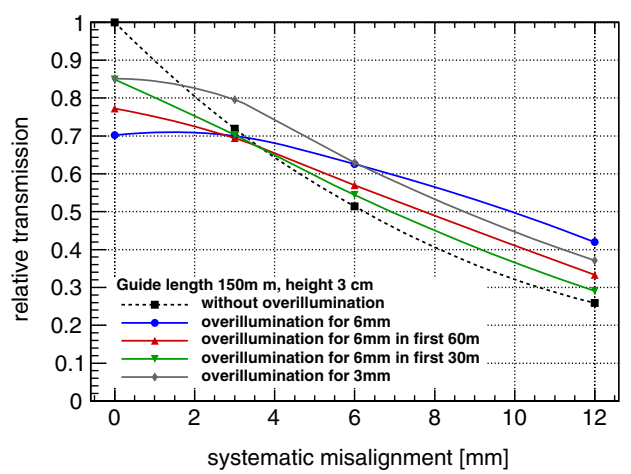

(a)

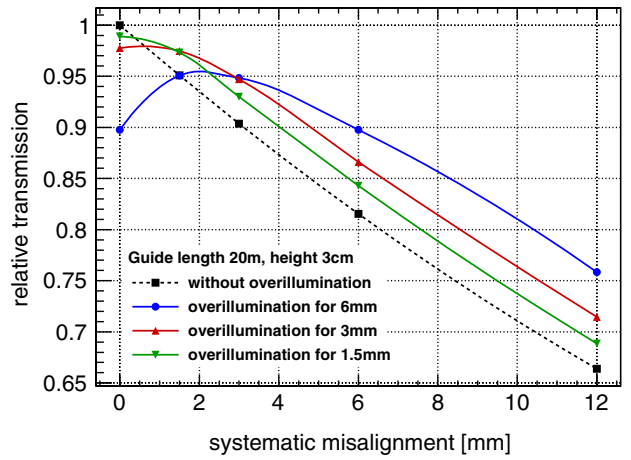

(b)

FIG. 3. Relative transmission with respect to perfectly aligned guide, with different amounts of systematic vertical misalignment and overillumination for a certain expected misalignment, $150 \mathrm{~m}$ guide (a) and $20 \mathrm{~m}$ guide (b) with $1 \mathrm{~m}$ long segments. Only systematic misalignment. 
section(s) only, where the systematic effect is largest. The green and red lines in Fig. 3(a) (downwards and upwards triangular markers) correspond to an overillumination in the first $30 \mathrm{~m}$ or $60 \mathrm{~m}$, respectively. The loss from overillumination is significantly reduced, but so is the gain in the case of misalignment. A better strategy is to employ overillumination throughout the whole length of the guide, but with an optimistic misalignment expectation: The gray line (diamond shaped markers) in Fig. 3(a) shows that the performance with an overillumination designed for a $3 \mathrm{~mm}$ shift is the same as with the $6 \mathrm{~mm}$ expectation for a "real" (simulated) misalignment of $6 \mathrm{~mm}$, and much better for smaller systematic shifts.

Figure 3(b) follows this strategy further for the $20 \mathrm{~m}$ long guide example, comparing overilluminations designed for 6,3 and $1.5 \mathrm{~mm}$. The results are slightly different from those of the long guide: for a short guide with a certain systematic misalignment, the same relative transmission can be achieved by an overillumination designed for a misalignment value that is equal or twice the expectation. In case of short beam lines, a pessimistic alignment expectation seems to be a better approach: with a systematic misalignment between 4 and $5 \mathrm{~mm}$, the highest relative transmission in a $20 \mathrm{~m}$ long guide is obtained with the $6 \mathrm{~mm}$ overillumination, while the $150 \mathrm{~m}$ guide shows the best performance with the $3 \mathrm{~mm}$ overillumination. On the other hand, the systematic misalignment values correspond to the maximum misalignment, reached over a considerable period of time. Therefore, an optimistic overillumination design might be suitable for short instruments after all, to ensure a good performance right at the beginning of operations.

The comparison between results for the long and short instruments implies that the losses from overillumination increase more rapidly with the number of guide segments than the losses from misalignment. The ideal solution has to be evaluated for each instrument separately, taking the instrument's length as well as a possible realignment schedule or staffing plan into account.

When the random misalignment is included in Fig. 4, the relative performance with different amounts of overillumination is the same while the transmission without any overillumination is more strongly decreased. We therefore conclude that there are overillumination approaches that always perform better than no overillumination for a guide with constant cross section: an overillumination design based on $3 \mathrm{~mm}$ or less seems to be advantageous.

Note, however, that even with no systematic misalignment, the loss from purely random misalignment in a $150 \mathrm{~m}$ long guide in Fig. 4(a) is already more than $30 \%$. We therefore anticipate that measures to realign the guide during operations should be foreseen.

\section{MODEL SENSITIVITY TO VARIATIONS}

The shape of the systematic misalignment in Eq. (1) has been designed from SNS experience combined with the planned ESS building and shielding layout, but details such as the tilt of the cosine axis are difficult to predict. Whilst the uncertainty in the amplitude, i.e. the amount of ground movement, is covered by the comparison of different overillumination designs with different simulated guide shifts in the previous section, uncertainties in the shape of function (1) are evaluated in Fig. 5 for the $150 \mathrm{~m}$ long guide. Deviations of $20 \%$ from the assumed model are tested. Only systematic misalignment without random contributions is simulated here. We used overillumination designed for an expected ground movement of $3 \mathrm{~mm}$ based on the results of Figs. 3(a) and 4(a).

If the ground deformations oscillate with a $20 \%$ higher frequency than modeled, the relative transmission for a $3 \mathrm{~mm}$ maximum movement is about $4 \%$ less than expected.

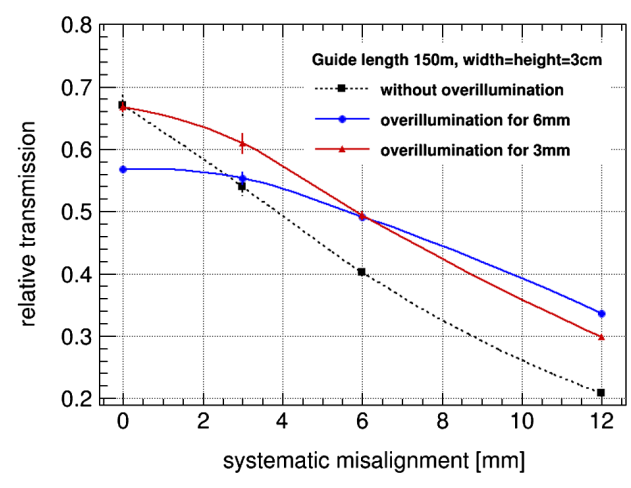

(a)

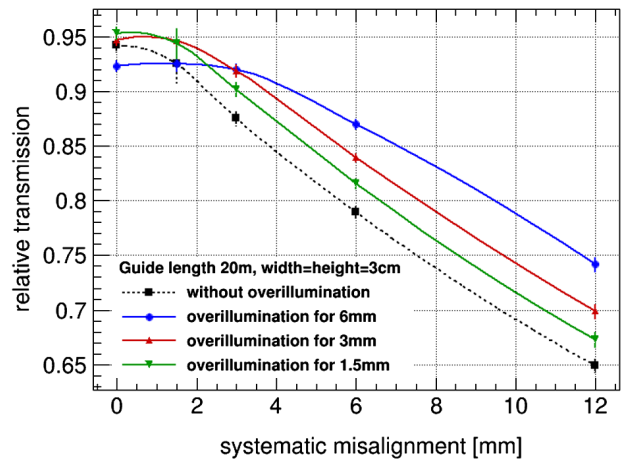

(b)

FIG. 4. Relative transmission with respect to perfectly aligned guide, with different amounts of systematic vertical misalignment and overillumination for a certain expected misalignment, $150 \mathrm{~m}$ guide (a) and $20 \mathrm{~m}$ guide (b) with $1 \mathrm{~m}$ long segments. Systematic plus random misalignment using the mean of five random misalignment sets. Note that the $y$ axes are suppressed and have different scales in each graph. 


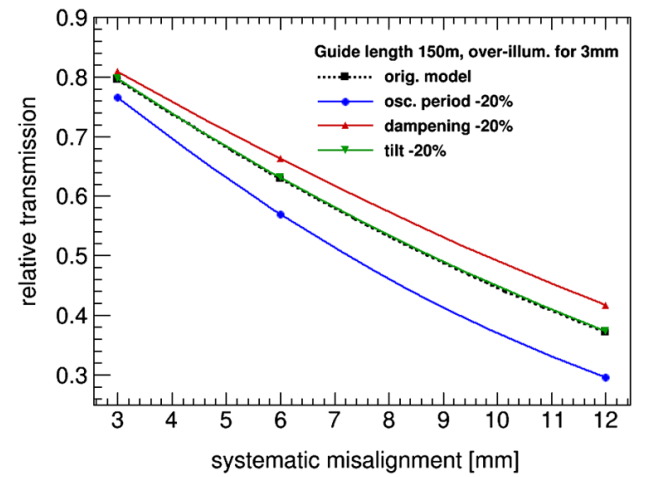

FIG. 5. Estimation of systematic uncertainty of the relative transmission with overillumination, assuming modifications to Eq. (1). Relative transmission is plotted against amplitude of systematic misalignment, assuming a $3 \mathrm{~mm}$ overillumination, with the original function, a $20 \%$ change variation in the period of the damped cosine, the same $20 \%$ change in the damping factor, and also in the tilt.

This difference increases to $6 \%$ at $6 \mathrm{~mm}$. A $20 \%$ weaker dampening of the oscillations leads to a smaller difference from the predicted behavior, and a $20 \%$ weaker tilt gives almost the same performance as expected from the model in function (1). The systematic uncertainty on the performance with overillumination to mitigate a systematic ground movement can hence be estimated to be around $5 \%$ for the period and dampening contribution, and negligible for the tilt contribution. A $20 \%$ variation of the amplitude also leads to relative transmissions within 5\% of the expectations. Neglecting the tilt and assuming the three components with 5\% each to be independent, a total systematic uncertainty of $10 \%$ is anticipated.

\section{CONCLUSIONS}

Systematic guide misalignment, caused by ground deformations, has been found to be a significant expected contributor to instrument performance losses at the ESS.

We have presented a mitigation strategy, based on overillumination. Losses from overillumination increase more rapidly with the number of guide segments than the losses from misalignment, therefore the best mitigation strategy, in particular for long guides, is to apply overillumination for an optimistic misalignment that is roughly half of the pessimistic/conservative level.

Finally, we estimate that our empirical model is sensitive to functional variations in the ground deformation, leading to approximately $10 \%$ variations in the predicted performance reductions.

\section{ACKNOWLEDGMENTS}

This work greatly benefited from SNS data kindly shared by K. Herwig and J. Error.
[1] F. Mezei, The raison d'être of long pulse spallation sources, J. Neutron Res. 6, 3 (1997).

[2] P. Böni, New concepts for neutron instrumentation, Nucl. Instrum. Methods Phys. Res., Sect. A 586, 1 (2008).

[3] K. H. Klen $\varnothing$, K. Lieutenant, K. H. Andersen, and K. Lefmann, Systematic performance study of common neutron guide geometries, Nucl. Instrum. Methods Phys. Res., Sect. A 696, 75 (2012).

[4] C. Zendler, D. Nekrassov, and K. Lieutenant, An improved elliptic guide concept for a homogeneous neutron beam without direct line of sight, Nucl. Instrum. Methods Phys. Res., Sect. A 746, 39 (2014).

[5] L. D. Cussen, D. Nekrassov, C. Zendler, and K. Lieutenant, Multiple reflections in elliptic neutron guide tubes, Nucl. Instrum. Methods Phys. Res., Sect. A 705, 121 (2013).

[6] Y. Kawabata, S. Suzuki, M. Sakamoto, T. Harami, H. Takahashi, and N. Onishi, Transmission efficiency of neutron guide tube with alignment errors, J. Nucl. Sci. Technol. 27, 406 (1990).

[7] Y. Kawabata, M. Suzuki, H. Takahashi, N. Onishi, A. Shimanuki, Y. Sugawa, N. Niino, T. Kasai, K. Funasho, S. Hayakawa, and K. Okuhata, Construction of neutron guide tubes in upgraded jrr-3, J. Nucl. Sci. Technol. 27, 1138 (1990).

[8] Y. Kawabata, M. Suzuki, H. Kikuchi, and M. Sano, Characteristics of neutron guide tubes in upgraded jrr-3, J. Nucl. Sci. Technol. 30, 1050 (1993).

[9] P. M. Allenspach, P. Böni, and K. Lefmann, Loss mechanisms in supermirror neutron guides, Proc. SPIE Int. Soc. Opt. Eng. 4509, 157 (2001).

[10] W.-T. Lee and F. Klose, SNS magnetism reflectometer: Basic design and neutron guide optimization using Monte Carlo simulations, Proc. SPIE Int. Soc. Opt. Eng. 4509, 145 (2001)..

[11] Possible guide vendor (private communication).

[12] R. Ryan, Challenges of neutron guide alignment at the NIST Center for Neutron Research, 2011, https://www .eastcoastmetrology.com/pdf/NCNR.pdf.

[13] A. J. Jackson and K. Kanaki, ESS Construction Proposal LoKI-A broad-band SANS instrument, LoKI instrument proposal.

[14] E. Oksanen, ESS instrument construction proposal macromolecular diffractometer, NMX instrument proposal.

[15] C. Zendler, D. M. Rodriguez, and P. M. Bentley, Generic guide concepts for the European Spallation Source, Nucl. Instrum. Methods Phys. Res., Sect. A 803, 89 (2015).

[16] H. Möller, 2015, ESS CF Report No. G01-DT-DEFSGDGeoPM14_ConcretePiles.

[17] K. W. Herwig and J. Error (private communication).

[18] K. Lieutenant, G. Zsigmond, S. Manoshin, M. Fromme, H. N. Bordallo, D. Champion, J. Peters, and F. Mezei, Neutron instrument simulation and optimization using the software package VITESS, Proc. SPIE Int. Soc. Opt. Eng. 5536, 134 (2004).

[19] C. Zendler, K. Lieutenant, D. Nekrassov, and M. Fromme, VITESS 3-virtual instrumentation tool for the European Spallation Source, J. Phys. Conf. Ser. 528, 012036 (2014).

[20] S. Peggs et al., 2013, European Spallation Source Technical Design Report No. ESS-2013-001, ISBN 978-91-980 173-2-8. 